\title{
Avaliação de diferentes armações no recolhimento mecanizado do amendoim
}

Submetido - 01 ago. $2020 \quad$ Aprovado - 04 set. $2020 \quad$ Publicado - 14 out. 2020
dol http://dx.doi.org/10.17648/sas.v1i1.89

\section{Armando Lopes de Brito Filho (D)}

Mestrando do Programa de Pós-Graduação em Agronomia (Ciência do Solo), Universidade Estadual Paulista, Campus Jaboticabal, e-mail: armando.brito@unesp.br.

Franciele Morlin Carneiro

Pós-Doutoranda do Programa de Pós-Graduação em Agronomia (Produção Vegetal), Universidade Estadual Paulista, Campus Jaboticabal, e-mail: franciele.morlin@unesp.br.

Jarlyson Brunno Costa Souza iD

Mestrando do Programa de Pós-Graduação em Agronomia (Produção Vegetal), Universidade Estadual Paulista, Campus Jaboticabal, e-mail: jarlyson.brunno@unesp.br.

\section{Samira Luns Hatum de Almeida (iD}

Doutoranda do Programa de Pós-Graduação em Agronomia (Produção Vegetal), Universidade Estadual Paulista, Campus Jaboticabal, e-mail: samira.lh.almeida@unesp.br.

\section{Jean Lucas Pereira Oliveira iD}

Doutorando do Programa de Pós-Graduação em Agronomia (Produção Vegetal), Universidade Estadual Paulista, Campus Jaboticabal, e-mail: jlp.oliveira@unesp.br.

Prof. Dr. Livre-Docente da UNESP/FCAV (Produção Vegetal), Jaboticabal-SP, e-mail: rouverson.silva@unesp.br.

\section{RESUMO}

Com o aumento do crescimento populacional e para atender a demanda, é de suma importância procurar a obtenção de maiores produtividades, e um dos fatores que afeta bastante a produtividade são perdas oriundas da colheita, sendo que, as mesmas precisam ser reduzidas quando detectadas. Desta maneira objetivou-se, com este trabalho, quantificar as perdas em função do tipo de armação, durante o recolhimento do amendoim, e comparar qual armação foi a melhor para determinação das perdas. $O$ experimento foi conduzido na safra 2019/2020, no município de Ribeirão Preto, estado de São Paulo. A área experimental foi instalada em sistema de Meiosi. O delineamento experimental foi em faixas contendo 2 tratamentos (armação circular e retangular) e 20 repetições, perfazendo 40 parcelas. Cada parcela possuiu o distanciamento de $80 \mathrm{~m}$ de comprimento entre si. A variável analisada foi perda total, que foi obtida pelo o somatório das perdas visíveis e invisíveis, que foi coletada durante o recolhimento. Após a coleta dos dados foi efetuado teste $F$ e quando significativo foi usado o teste de Tukey. Através da análise estatística verificou-se alta variabilidade nos dados de perdas, um fato comum quando se trabalha com perdas e colheita mecanizada, encontrando-se diferença estatística na comparação das perdas das diferentes armações, no qual a armação circular apresentou valores mais confiáveis devido ao fato de possuir uma representatividade maior da área.

Palavras-chave: Arachis hypogaea L.; Perdas quantitativas; Produtividade.

\section{Evaluation of different frames in mechanized peanut collection}

\section{ABSTRACT}

With the increase in population growth and to meet the demand, it is extremely important to seek to obtain higher productivity, and one of the factors that greatly affects productivity are 
losses arising from the harvest, and they need to be reduced when detected. In this way, the objective of this work was to quantify the losses according to the type of frame, during the peanut collection, and to compare which frame was the best for determining the losses. The experiment was conducted in the 2019/2020 harvest, in the municipality of Ribeirão Preto, state of São Paulo. The experimental area was installed in a Meosi system. The experimental design was in bands containing 2 treatments (circular and rectangular frame) and 20 repetitions, totaling 40 plots. Each plot was $80 \mathrm{~m}$ long apart. The analyzed variable was total loss, which was obtained by the sum of visible and invisible losses, which was collected during collection. After data collection, an F test was performed and when significant, the Tukey test was used. Through statistical analysis, there was a high variability in the loss data, a common fact when working with losses and mechanized harvesting, finding a statistical difference when comparing the losses of the different frames, in which the circular frame presented more reliable values due to the fact of having a greater representativeness of the area.

Keywords: Arachis hypogaea L.; Quantitative losses; Productivity.

\title{
Evaluación de diferentes marcos en la recolección mecanizada de maní
}

\begin{abstract}
RESUMEN
Con el aumento del crecimiento de la población y para satisfacer la demanda, es extremadamente importante tratar de obtener una mayor productividad, y uno de los factores que afecta en gran medida la productividad son las pérdidas derivadas de la cosecha, y deben reducirse cuando se detectan. De esta manera, el objetivo de este trabajo fue cuantificar las pérdidas según el tipo de marco, durante la recolección de maní, y comparar qué marco era el mejor para determinar las pérdidas. El experimento se realizó en la cosecha 2019/2020, en el municipio de Ribeirão Preto, estado de São Paulo. El área experimental se instaló en un sistema Meosi. El diseño experimental fue en bandas que contenían 2 tratamientos (marco circular y rectangular) y 20 repeticiones, totalizando 40 parcelas. Cada parcela tenía $80 \mathrm{~m}$ de largo de separación. La variable analizada fue la pérdida total, que se obtuvo por la suma de las pérdidas visibles e invisibles, que se recolectó durante la recolección. Después de la recolección de datos, se realizó una prueba $F y$, cuando fue significativa, se utilizó la prueba de Tukey. A través del análisis estadístico, hubo una gran variabilidad en los datos de pérdidas, un hecho común cuando se trabaja con pérdidas y cosecha mecanizada, encontrando una diferencia estadística al comparar las pérdidas de los diferentes cuadros, en los que el cuadro circular presenta valores más confiables debido a hecho de tener una mayor representatividad de la zona.
\end{abstract}

Palabras clave: Arachis hypogaea L.; Pérdidas cuantitativas; Productividad.

\section{Introdução}

Em virtude do aumento do crescimento populacional têm-se a preocupação e a necessidade de melhorias no sistema agrícola, para que a demanda de alimentos atenda ao consumo alimentício, visando pela maior produtividade e redução de perdas durante a colheita.

Para a produção do amendoim, a colheita é tida como uma das operações críticas, devido a presença de perdas, que são inevitáveis, que pode chegar até à 30\% da produtividade (SANTOS et al., 2013). No Brasil, a maior parte da produção de amendoim concentra-se no estado de São Paulo, na região de Ribeirão Preto, onde esta cultura normalmente é implantada em rotação e sucessão de cultura, reformas de canaviais 
(BARBOSA; HOMEM; TARSITANO, 2014) ou em sistema de Meiosi Método Inter Ocupacional Simultâneo.

Vendo a importância para a redução dessas perdas é relevante quantificar o quanto se perde, que é feito por meio de armações. Devido ao desenvolvimento das vagens serem sob o solo há presença de perdas visíveis e invisíveis, o que se torna mais difícil de saber o quanto se teve de perdas quando não se quantifica de forma adequada. Normalmente os fatores que influenciam nas perdas são desfolha prematura, excesso de maturação, solo seco e/ou compactado, e pedúnculo enfraquecido (SILVA, 2019).

Com isso este trabalho, teve como objetivo quantificar as perdas em função do tipo de armação (retangular e circular) durante o recolhimento do amendoim, e comparar qual armação foi a melhor para determinação das perdas.

\section{Material e métodos}

O experimento foi conduzido na safra 2019/2020, no município de Ribeirão Preto - SP, situado nas coordenadas geográficas $21^{\circ} 20^{\prime} 17.55^{\prime \prime S}$ e 4754'7.31"O (Figura 1A). A área experimental foi instalada em sistema de Meosi, que pode ser observado na Figura 1B. Esta área possui solo Latossolo Vermelho e o clima da região é Aw, tropical com inverno seco, conforme a classificação climática de Köppen (ALVARES et al., 2013).

A cultivar semeada foi a IAC OL3, onde foram distribuídas 18 sementes $\mathrm{m}^{-1}$ com o espaçamento entre linhas de 0,90 m. NA semeadura foi utilizado o trator John Deere 4x2 TDA, modelo $7195 \mathrm{~J}$, acoplado à semeadora Jumil, modelo JM 2980 PD com 6 linhas de semeadura.

O recolhimento foi efetuado três dias após o arranquio, onde foi usado o trator John Deere 4x2 TDA, modelo $7195 \mathrm{~J}$, acoplado na recolhedora de marca Miac, modelo Twin Master Tripla, com largura da plataforma 5,40 m. A velocidade média de trabalho foi mantida entre 3,8 km $\mathrm{h}^{-1}$. O delineamento experimental foi em faixas contendo 2 tratamentos 
(armação circular e retangular) e 20 repetições, perfazendo 40 parcelas. Cada parcela possuiu o distanciamento de $80 \mathrm{~m}$ de comprimento entre si.

Quanto as armações circulares foram quatro, sendo que, cada uma possui dimensão de $0,25 \mathrm{~m}^{2}$, totalizando $1 \mathrm{~m}^{2}$ (Figura 2A). A armação de formato retangular possuiu 5,4 $\mathrm{m}$ de comprimento por $0,37 \mathrm{~m}$ de largura (Figura 2 B). As armações circulares foram arremessadas entre os rodados da recolhedora e a retangular foi colocada após a passagem da máquina.

A variável analisada foi perda total, que foi obtida pelo o somatório das perdas visíveis e invisíveis, que foi coletada durante o recolhimento. Após a coleta dos dados foi efetuado a análise descritiva e a comparação de média pelo teste $F$, quando significativo foi usado o teste de Tukey a $5 \%$ de significância.

A.

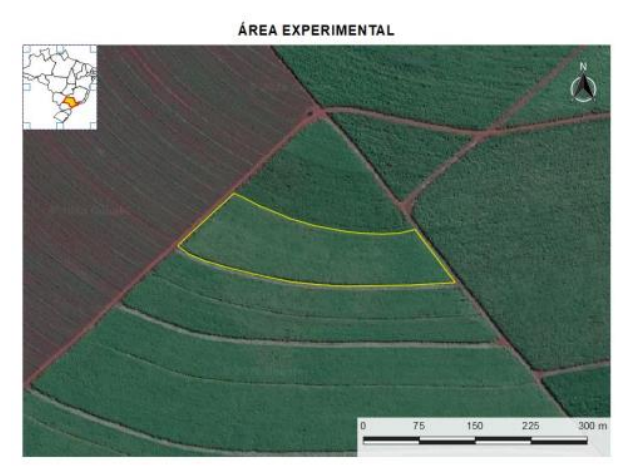

B.

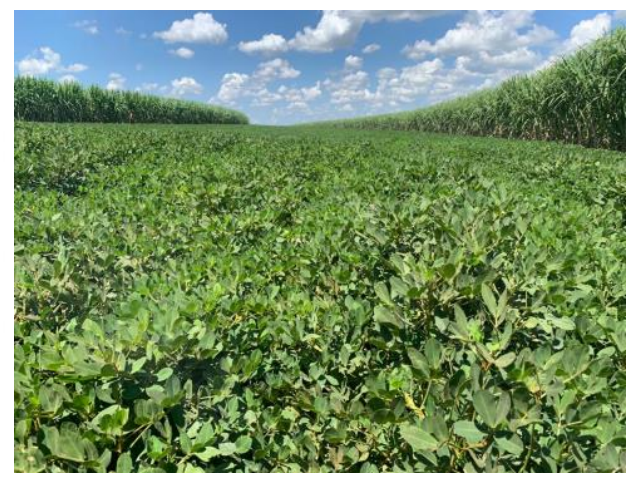

Figura 1. Localização da área experimental (A.) e o local onde a cultura foi implantada, que foi no sistema de Meiosi (B.).

A.

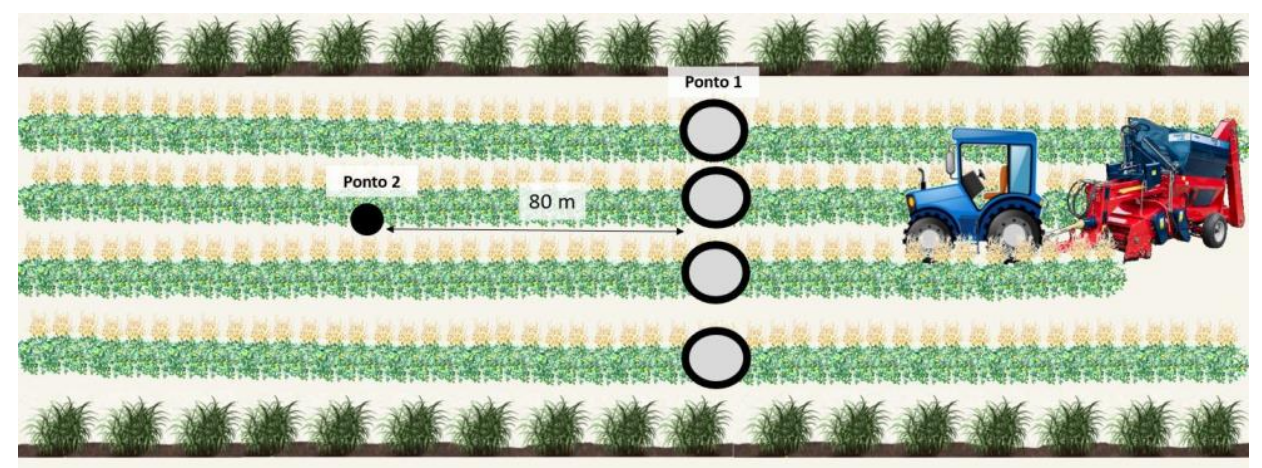

B. 


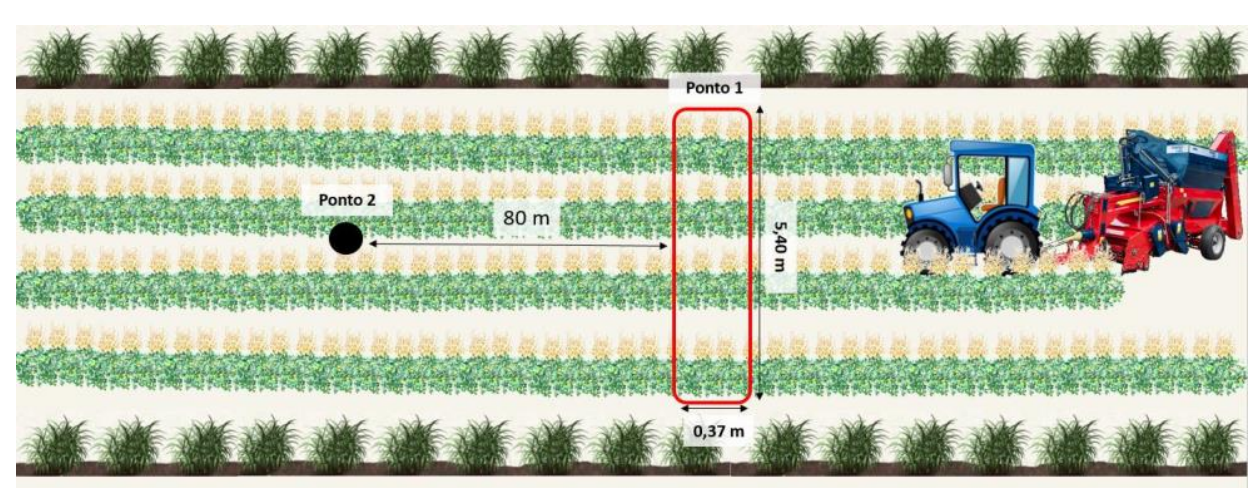

Figura 2. Armações circulares (A.) e retangulares (B.) utilizadas pa। coleta de perdas quantitativas.

\section{Resultados e discussão}

Pela comparação das médias das armações (Tabela 1) observou-se que houve efeito significativo entre os tratamentos $(p<0,05)$, no qual a armação circular apresentou valores bem acima da armação retangular, podendo ser devido ao fato da armação circular apresentar uma maior quantidade de pontos amostrados, pois foi utilizado quatro aros, desse modo resultando uma maior representatividade.

Tabela 1. Utilização de diferentes armações para quantificação da perda total $\left(\mathrm{kg} \mathrm{ha}^{-1}\right)$ na colheita de amendoim.

\begin{tabular}{ccc}
\hline \multirow{2}{*}{ Variável } & \multicolumn{2}{c}{ Armação } \\
\cline { 2 - 3 } & Circular & Retangular \\
\hline Perdas Totais $\left(\mathrm{kg} \mathrm{ha}^{-1}\right.$ & $368,37 a$ & $216,93 \mathrm{~b}$ \\
\hline Letras diferentes na linha diferem entre si pelo teste de Tukey a 5\% de probabilidade.
\end{tabular}

$\mathrm{Na}$ análise descritiva (Tabela 2) dos dados, verificou-se pelo teste Ryan-Joiner distribuição normal. Para ambos tratamentos, o coeficiente de assimetria (Cs) foi observado distribuição assimétrica à direita com intensidade moderada $(0,15<|\mathrm{Cs}|<1)$, quanto ao coeficiente de curtose os dados apresentaram distribuição platicúrtica com uma curva mais achata, devido a dispersão dos valores em relação à média. E essa dispersão também foi observada para o coeficiente de variação (CV), de forma que os valores tiveram alta variabilidade. Um padrão comum de acordo com a literatura na avaliação de perdas, com valores de CV na maioria dos estudos superiores a $30 \%$. 
Tabela 2. Análise descritiva da perda total $\left(\mathrm{kg} \mathrm{ha}^{-1}\right)$ para as armações circular e retangular.

\begin{tabular}{lrrrrrr}
\hline \multirow{2}{*}{ Armação } & \multicolumn{6}{c}{ Perda Total $\left(\mathrm{kg} \mathrm{ha}^{-1}\right)$} \\
\cline { 2 - 7 } & $\overline{\mathrm{X}}$ & $\sigma$ & $\mathrm{CV}(\%$ & Cs & Ck & RJ \\
\cline { 2 - 7 } Circular & 368,3 & 207,61 & 56,37 & 0,62 & $-0,55$ & 0,975 \\
Retangular & 216,$9 ;$ & $67,4 C$ & $31,0 \subseteq$ & 0,25 & $-0,77$ & 0,987 \\
\hline
\end{tabular}

$\bar{X}$ - média geral; $\sigma$ - desvio padrão; CV (\%) - Coeficiente de variação; Cs - coeficiente de assimetria; Ck - coeficiente de curtose; RJ - teste de normalidade por Ryan-Joiner ( $\mathrm{N}$ Normal; $A$ - não normal $p<0.10)$.

Esses altos valores do CV encontrados, são inerentes a vários fatores presentes no campo, como a condução da operação, relação máquina-planta-solo, clima, entre outros que influenciam o sistema de colheita.

Assim como demonstram os autores Zerbato et a. (2017) trabalhando com amendoim, Cortez et al. (2019) na soja, Toledo et al. (2008) com cana-de-açúcar, que independente da cultura estudada, trabalhos com colheita mecanizada estão sujeitos a diversos fatores não controláveis que afetam a variabilidade do processo.

Desta maneira o maior valor apresentado pela armação circular pode ser associado a maior representatividade da área de colhida, por coletar amostras em locais diferentes o que resulta em maior confiabilidade dos dados e, consequentemente, melhor representação dos índices de perdas totais.

\section{Conclusões}

Houve diferença entre a mensuração de perdas das diferentes armações. No qual a armação circular apresentou maior confiabilidade na obtenção dos dados, por apresentar uma maior representatividade da área.

\section{Agradecimentos}

A Coordenação de Aperfeiçoamento de Pessoal de Nível Superior Brasil (CAPES) - Código de Financiamento 001, pela concessão da bolsa de mestrado do primeiro autor. 


\section{Referências}

ALVARES, Clayton Alacarde et al. Köppen's climate classification map for Brazil. Meteorologische Zeitschrift, Stuttgart, v. 22, n. 6, p.711-728, 2013. https://doi.org/10.1127/0941-2948/2013/0507

BARBOSA, Rafael Marani; HOMEM, Bruno Fernandes Modesto; TARSITANO, Maria Aparecida Anselmo. Custo de produção e lucratividade da cultura do amendoim no município de Jaboticabal, São Paulo. Revista Ceres, Viçosa, v. 61, n. 4, p. 475-481, 2014. http://dx.doi.org/10.1590/0034$\underline{737 \times 201461040005}$

CORTEZ, Jorge W.; SYRIO, Marina G.; RODRIGUES, Sonia A. Types of header, operating speed, and geometry of collection frames on the total losses of soybean harvest. Engenharia Agrícola, 2019, 39.4: 482-489. http://doi.org/10.1590/1809-4430-eng.agric.v39n4p482-489/2019

SANTOS, Edvaldo Pereira dos et al. Produtividade e perdas de amendoim em cinco diferentes datas de arranquio. Revista Ciência Agronômica, Fortaleza, v. 44, n. 4, p. 695-702, 2013.

SILVA, Rouverson Pereira da. Colheita mecanizada de amendoim. In: SILVA, Rouverson Pereira da; SANTOS, Adão Felipe dos; CARREGA, Willians César (ed.). Avanços na produção de amendoim. Jaboticabal: Funep, 2019. p. 129-146.

TOLEDO, Anderson de. et al. Caracterização de perdas e distribuição de vegetação em soja mecanizada de colheita. Engenharia Agrícola 28(4):710-719, 2008.

ZERBATO, Cristiano et al. Statistical control of processes aplied for peanut mechanical digging in soil textural classes. Engenharia Agrícola 37(2): 315322, 2017. DOI: http://dx.doi.org/10.1590/1809-4430-eng.agric.v37n2p315$\underline{322 / 2017}$ 